\title{
Modelos de retornos esperados no mercado brasileiro: testes empíricos utilizando metodologia preditiva
}

\author{
Adriano Mussa ${ }^{1}$ \\ Pablo Rogers ${ }^{2}$ \\ José Roberto Securato ${ }^{3}$
}

\section{Resumo}

Metodologias preditivas para teste de modelos de retornos esperados são amplamente difundidas no meio acadêmico internacional, entretanto, não têm sido sistematicamente aplicadas no Brasil. Geralmente, os estudos empíricos procedidos com dados do mercado acionário brasileiro concentram-se apenas na primeira etapa dessas metodologias. O objetivo deste artigo foi testar e comparar os modelos CAPM (Capital Asset Pricing Model), 3-fatores e 4-fatores a partir de uma metodologia de teste preditivo, utilizando duas etapas - regressões em séries temporais e cross-section - com erros-padrão calculados pela técnica de Fama e Macbeth (1973). Apesar dos resultados indicarem a superioridade do modelo 4fatores em relação ao modelo 3-fatores, e esse em relação ao CAPM, nenhum dos modelos testados foram suficientes na explicação das variações dos retornos das ações do mercado brasileiro. Contrário a algumas evidências empíricas que não utilizam a metodologia preditiva, os efeitos tamanho e momento parecem não estar presentes no mercado de capitais brasileiro, enquanto há indícios da presença do efeito valor e relevância do mercado em explicar retornos esperados. Os achados dessa pesquisa levantaram alguns questionamentos, principalmente, devido à originalidade metodológica no mercado nacional e o tema ser ainda incipiente e polêmico no meio acadêmico brasileiro.

Palavras-chave: CAPM. Efeito Tamanho. Efeito Momento. Efeito Valor.

${ }^{1}$ Doutorando em Administração (Finanças) pela FEA/USP. Mestrado em Administração. Pontifícia Universidade Católica de São Paulo, PUC/SP. Professor da Pontifícia Universidade Católica de São Paulo, PUC/SP. Endereço: Rua Apiacás, 62, Apto. 182, Perdizes, São Paulo, SP, 05017-020. Email:adriano.mussa@labfin.com.br.

${ }^{2}$ Doutorando em Administração (Finanças) pela FEA/USP. Professor Assistente da Universidade Federal de Uberlândia Endereço: Rua José Miguel Saramago, 1168, Apto. 301, Santa Mônica, Uberlândia, MG, 38400-222. E-mail: pablo@fagen.ufu.br.

${ }^{3}$ Livre-Docente em Administração pela FEA/USP. Professor Titular do Departamento de Administração da FEA/USP. Endereço: Rua Navarro de Andrade, 152, Pinheiros, São Paulo, SP, 05418-020. E-mail: securato@usp.br. Artigo recebido em: 22/02/2008. Aceito em: 30/07/2008. Membro do Corpo Editorial Científico responsável pelo processo editorial: Rolando Juan Soliz Estrada. 


\section{Introdução}

Marco para os modelos de precificação de ativos foi o trabalho de Sharpe (1964) que, tendo como base as pesquisas de Markowitz (1952) e de Tobin (1958), desenvolveu o famoso modelo denominado Capital Asset Pricing Model (CAPM). Esse é um modelo de fator único: a partir de uma relação apenas, o beta explica a diferença de retorno exigido entre os ativos. Entretanto, apesar de ser um modelo simples e lógico, o desenvolvimento dos testes do CAPM trouxe o debate sobre suas deficiências, principalmente devido ao surgimento de novas evidências de que boa parte da variação nos retornos esperados dos ativos não está relacionada ao fator beta de mercado (FAMA; FRENCH, 2004).

Nesse sentido, Ross (1976) propôs um novo enfoque multifatorial para explicar a formação de preços dos ativos, baseado nos conceitos de arbitragem, dando origem ao Arbitrage Pricing Theory (APT). Grinblatt e Titman (2005) destacam que existem três maneiras de estimar os fatores comuns em um modelo multifatorial: 1) procedimento estatístico para determinar as carteiras fatoriais, criadas para imitar fatores; 2) utilizar variáveis macroeconômicas na condição de aproximação para fatores; 3) utilizar características da empresa, como o seu tamanho, para criar carteiras que ajam como aproximações para os fatores. Essa terceira opção utiliza carteiras selecionadas com base nas anomalias de retornos passados. Basu (1977), Banz (1981), Stattman (1980), Lakonishok e Shapiro (1986) e Fama e French (1992) são exemplos de alguns estudos que identificaram ineficiências do CAPM e terminaram por caracterizar algumas anomalias de mercado.

$\mathrm{Na}$ busca por fatores que pudessem melhorar o poder explicativo do CAPM e capturar anomalias do mercado, Fama e French (1993) formularam o modelo denominado 3- fatores. Esse modelo, segundo os autores, poderia explicar significativamente os retornos das ações. Os fatores de risco são o mercado, o tamanho da empresa e o índice book-to-market ou B/M. Uma vez identificado o fator de risco momento por Jegadeesh e Titman (1993), alguns estudos, sendo pioneiro o trabalho de Carhart (1997), passaram a adicioná-lo ao modelo 3-fatores de Fama e French (1993), construindo o que ficou conhecido como modelo 4-fatores. Em seu estudo, Carhart (1997) encontrou evidências empíricas para afirmar a superioridade do modelo 4fatores em relação ao modelo 3-fatores na explicação dos retornos. 
Alguns estudos também foram efetuados no mercado acionário brasileiro. Málaga e Securato (2004) corroboraram a validade do modelo 3-fatores de Fama e French (1993) e sua superioridade em relação ao CAPM. Mussa, Santos e Famá (2007), por sua vez, testaram o modelo 4-fatores de Carhart (1997), também o validando e chegando a evidências de sua superioridade em relação aos modelos CAPM e 3-fatores. No trabalho de Fama e French (1993), assim como em ambas as pesquisas brasileiras, foram utilizadas regressões em séries temporais para teste e validação dos modelos, sendo que o uso desta técnica estatística se constitui em uma das mais importantes limitações dessas pesquisas. Aliás, tais limitações foram destacadas pelos próprios autores.

Conforme Lakonishok e Shapiro (1986), testes que utilizam unicamente regressões em séries temporais são conhecidos como contemporâneos; adotam o mesmo período para estimar tanto as variáveis explicativas quanto a relação entre elas e a variável dependente; têm a desvantagem de superestimar a importância e a significância das variáveis explicativas, quando comparados a modelos de testes preditivos que utilizam períodos diferentes para a estimação das variáveis explicativas, e a relação delas com a variável dependente. Além disso, testes preditivos tendem a refletir mais adequadamente o comportamento dos investidores, uma vez que estes utilizam dados históricos para a estimação dos betas, com o objetivo de realizar seus investimentos futuros.

Vários procedimentos de testes do CAPM e modelos relacionados são discutidos por Elton et al. (2004). Metodologia interessante de teste preditivo é a de Fama e Macbeth (1973), que realizaram os testes empíricos do CAPM por meio de dois passos: o primeiro passo, semelhante ao realizado por Fama e French (1993), consistiu em realizar regressões em séries temporais com dados da amostra within-sample, que serviram para a estimação dos betas dos fatores de risco; o segundo, utilizou esses betas estimados como variáveis explicativas em regressões cross-section, com dados da amostra out-ofsample, com o intuito de testar as hipóteses resultantes dos modelos. Nesse passo, Fama e Macbeth (1973) utilizaram técnica própria no cômputo dos erros-padrão dos coeficientes.

Com base no exposto, o objetivo do presente trabalho é testar e comparar o poder de explicação dos modelos CAPM, 3-fatores e 4-fatores, utilizando um modelo de teste preditivo ao se aplicar a metodologia de regressões em duas etapas - séries temporais e cross-section - desenvolvida por 
Fama e Macbeth (1973). Adicionalmente é testada a hipótese de linearidade do CAPM. A amostra é constituída por todas as ações listadas na Bolsa de Valores do Estado de São Paulo - BOVESPA - de 1995 a 2007. Para dar maior robustez às análises, foram considerados diferentes períodos de withinsample e out-of-sample.

A pesquisa destaca-se no Brasil pela originalidade metodológica e por abordar um assunto importante para a comunidade acadêmica, à medida que agrega novas evidências a respeito de alguns dos modelos de precificação de ativos e fatores de risco mais discutidos na literatura internacional. O artigo está organizado da seguinte forma: a seção 2 aborda a fundamentação teórica do tema; a seção 3 explica a metodologia utilizada no estudo; a seção 4 aborda as análises dos resultados obtidos; e, finalmente, a seção 4 trata das considerações finais.

\section{Fundamentação Teórica}

\subsection{Arbitrage Pricing Theory (APT)}

O modelo APT, desenvolvido por Ross (1976), pode ser visto como um modelo mais genérico, uma alternativa ao CAPM. A principal diferença entre eles é que o APT pressupõe que os retornos dos ativos são gerados por uma série de fatores setoriais e gerais de mercado. Além disso, o APT exige hipóteses menos restritivas em relação ao comportamento do investidor.

As principais pressuposições do APT são, segundo Grinblatt e Titman (2005), apenas quatro: 1) os retornos podem ser descritos pelo modelo fatorial; 2) não há oportunidades de arbitragem; 3) existe um número amplo de ativos, de tal forma que é possível criar carteiras que diversifiquem o risco específico das empresas; 4) os mercados financeiros não possuem atrito.

Fama e French (2004) afirmam que, para modelos APT, o necessário é que as carteiras adicionais sejam bem diversificadas e que sejam suficientemente diferentes da carteira de mercado. Na mesma linha, Grinblatt e Titman (2005) destacam que a esperança é que o APT possa capturar aspectos adicionais de risco, além daqueles absorvidos pelo mercado, melhorando a explicação das variações dos retornos.

O modelo pode ser expresso na forma de retornos esperados, conforme a equação 1 (GRINBLATT; TITMAN, 2005, p. 180): 


$$
\text { (1) } \tilde{r}_{i}=\alpha_{i}+B_{i 1} * \tilde{F}_{1}+B_{i 2} * \tilde{F}_{2}+\ldots+B_{i K} * \tilde{F}_{K}+\tilde{\mathrm{E}}_{i}
$$

Onde $r_{i}=$ taxa aleatória de retorno do ativo $i ; a_{i}=$ taxa de retorno do ativo $i$, se todos os fatores de risco forem iguais a zero; $B_{i K}=$ a sensibilidade da taxa de retorno do ativo $i$ ao fator de risco $K ; F_{k}=$ fator de risco $K ; E_{i}=$ componente aleatório, com média zero e eliminado pela diversificação.

Da equação do APT, pode-se inferir, segundo Brealey e Myers (2003), que, ao se atribuir o valor zero aos indicadores de sensibilidade, o prêmio pelo ativo será nulo. Uma carteira diversificada que seja construída para não ter sensibilidade aos fatores macroeconômicos será, por essência, livre de risco, devendo oferecer retorno similar ao do ativo livre de risco. Caso oferecesse um retorno superior, os investidores poderiam obter retornos sem risco por meio de empréstimos de recursos à taxa livre de risco e aplicando tais recursos na carteira. Por outro lado, caso a carteira oferecesse um retorno inferior ao do ativo livre de risco, os investidores poderiam obter lucros sem risco, assumindo posições vendidas na carteira e compradas no ativo livre de risco. Estas posições são conhecidas como posições de arbitragem.

A pressuposição da equação do APT é que um número relativamente pequeno de fatores comuns, para os quais as ações têm sensibilidades distintas (betas), gera os retornos dos títulos. O erro da equação $\left(E_{i}\right)$ representa componentes de risco específicos das empresas, que não são correlacionados, contribuindo para uma variância mínima no caso de carteiras bem diversificadas (GRINBLATT; TITMAN, 2005). A magnitude dos betas do ativo descreve como se comporta a sensibilidade do retorno do título às mudanças dos fatores comuns. Ressalta-se que tanto o modelo 3-fatores como o modelo 4-fatores, objetos de estudo desta pesquisa, podem ser considerados modelos APT que utilizam características das empresas como fatores de risco.

\subsection{Modelo 3-fatores de Fama e French (1993)}

Fama e French (1993), com base nas anomalias identificadas em estudos anteriores, principalmente no estudo de Fama e French (1992), formularam um modelo de precificação de ativos de três fatores que, de acordo com os autores, explicaria de maneira significativa as variações dos retornos dos ativos. Os três fatores são: o risco de mercado, conforme definido por Sharpe 
(1964); o tamanho da empresa, que é medido pelo valor de mercado das ações e tenta capturar a anomalia efeito tamanho; e o índice book-to-market $(\mathrm{B} / \mathrm{M})$, que representa a relação entre o valor contábil e o de mercado do patrimônio líquido, e tenta capturar a anomalia denominada efeito valor. Os autores incluíram neste estudo tanto o mercado de bônus corporativo quanto o de ações.

Para a verificação da influência das variáveis sobre os retornos dos ativos, Fama e French (1993) criaram carteiras para replicar os fatores de risco relacionados às variáveis tamanho $e$ índice $\mathrm{B} / \mathrm{M}$. O primeiro passo foi estimar os prêmios pelos fatores de risco. O segundo foi analisar o efeito desses fatores sobre os retornos das ações por meio de regressões temporais. O período amostral para as ações foi de 1963 a 1991. A amostra foi composta por todas as ações listadas na NYSE (New York Stock Exchange), AMEX (American Stock Exchange) e NASDAQ (National Association of Securities Dealers Automated Quotations) (desta a partir de 1972).

O modelo pode ser expresso matematicamente por:

$$
\text { (2) } R_{c i, t}-R_{l r t}=a+b\left[R_{m t}-R_{l r t}\right]+s\left[S M B_{t}\right]+h\left[H M L_{t}\right]+e_{i, t}
$$

Onde $R_{C i, t}=$ retorno da carteira $i$, no mês $t ; R_{m t}=$ retorno da carteira de mercado no mês $t ; R_{\text {lrt }}=$ retorno do ativo livre de risco no mês $t ; S M B_{t}=$ prêmio pelo fator tamanho no mês $t$ (Small Minus Big ou a diferença entre a média de retorno das ações de empresas de pequeno porte e a média de retorno das ações de empresas de grande porte); $H M L_{t}=$ prêmio pelo fator $\mathrm{B} / \mathrm{M}$ no mês $t$ (High Minus Low ou a diferença entre a média de retorno das ações com alto índice $\mathrm{B} / \mathrm{M}$ e a média de retorno das ações com baixo índice $\mathrm{B} / \mathrm{M}) ; e_{i, t}=$ resíduo do modelo referente à carteira $i$ no mês $t$.

Os principais resultados do estudo de Fama e French (1993) são resumidos a seguir:

a) Foram encontrados prêmios positivos para todos os fatores de risco: mercado, tamanho e índice $\mathrm{B} / \mathrm{M}$;

b) A maior parte das regressões das carteiras do modelo apresentou intercepto estatisticamente igual a zero, validando o modelo utilizado e indicando que os três fatores seriam proxies para os fatores de risco relevantes para os investidores; 
c) Todos os fatores de risco mostraram-se significativos e pareceram se complementar na explicação das variações dos retornos das carteiras;

d) Os fatores de risco mostraram-se ortogonais, uma vez que a correlação entre eles apresentou-se baixa; $e$

e) O modelo dos três fatores é válido para ser usado nas diversas decisões financeiras e é superior ao CAPM na explicação dos retornos das ações no mercado norte-americano.

Como conseqüência da publicação do artigo de Fama e French (1993), muitos autores passaram a testar empiricamente a validade do modelo dos três fatores nos mais diversos mercados. Dentre os principais trabalhos realizados, tendo como objeto de estudo o mercado acionário brasileiro, pode-se citar Málaga e Securato (2004) e Lucena e Pinto (2005). Ambos encontraram evidências favoráveis à aplicação do modelo 3 -fatores no mercado acionário brasileiro, ou seja, as características da empresa, tamanho e índice book-to-market parecem explicar grande parte dos retornos das ações ao se utilizar a metodologia de regressões em séries temporais (primeiro passo da metodologia da presente pesquisa).

\subsection{Modelo 4-fatores}

Jegadeesh e Titman (1993) observaram a existência de um padrão de continuação dos retornos no mercado acionário norte-americano. Este padrão continuado significa a obtenção de retornos anormais positivos relacionados a uma estratégia baseada na análise do desempenho passado das ações. Mais especificamente, esta estratégia consiste em comprar as ações que tiveram bom desempenho passado e vender as ações que tiveram mau desempenho passado.

Grande número de estudos posteriores corroborou esta constatação, como Fama e French (1996) e Jegadeesh e Titman (2001) para o mercado norte-americano, Rouwenhorst (1998) para os mercados europeus e Rouwenhorst (1999) para os mercados emergentes. Fama e French (1996 e 2004) afirmam que o modelo 3 -fatores captura a maior parte das anomalias, 
com exceção do efeito momento. Carhart (1997), em seu estudo de análise da persistência de desempenho de fundos mútuos de investimento, agregou a estratégia de momento de Jegadeesh e Titman (1993) ao modelo 3-fatores de Fama e French (1993), construindo o que ficou conhecido como modelo 4-fatores.

Conforme Carhart (1997), o modelo 4-fatores é consistente com um modelo de equilíbrio de mercado, considerando a existência de quatro fatores de risco. $\mathrm{O}$ modelo pode ser entendido também como uma forma de APT que utiliza anomalias identificadas nos mercados como fatores de risco. Grinblatt e Titman (2005) destacam que modelos como este são compostos por carteiras de custo zero (ou seja, que se autofinanciam). Podem ser vistos como um modelo de atribuição de desempenho, no qual os coeficientes $e$ prêmios indicam a proporção do retorno que pode ser atribuído a: 1) uma posição comprada em carteira de índices de valores ponderados e uma posição vendida em ativos livres de risco; 2) uma posição comprada em uma carteira de ações de alto índice $\mathrm{B} / \mathrm{M}$ e uma posição vendida em ações com baixo índice $\mathrm{B} / \mathrm{M}$; 3) uma posição comprada em uma carteira de ações de baixa capitalização e uma posição vendida em uma carteira de ações de alta capitalização; e, finalmente, 4) uma posição comprada em uma carteira de ações que tiveram bom desempenho passado e uma posição vendida em uma carteira de ações que tiveram mau desempenho passado.

O modelo 4-fatores, composto pela adição do fator momento ao modelo 3-fatores de Fama e French (1993), pode então ser expresso pela equação 3 abaixo:

(3) $R_{c i, t}-R_{l r t}=a+b\left[R_{m t}-R_{l r t}\right]+s\left[S M B_{t}\right]+h\left[H M L_{t}\right]+w[$ WinMLost $]+e_{i, t}$

Onde, WinMLos $_{t}=$ prêmio pelo fator momento no mês $t$ (Winner Minus Loser ou a diferença entre a média de retorno das ações de empresas que tiveram um alto desempenho passado e a média de retorno das ações de empresas que tiveram um baixo desempenho passado).

Mussa, Santos e Fama (2007) aplicaram o modelo 4-fatores de Carhart (1997) no mercado acionário brasileiro, encontrando evidências de sua superioridade em relação aos modelos 3-fatores e CAPM, ao se utilizar regressões em séries temporais. 


\section{Metodologia}

\subsection{Composição da Amostra}

A amostra das ações analisadas foi composta por todas as ações listadas na BOVESPA entre $1^{\circ}$ de junho de 1995 e 30 de junho de 2007. Utilizou-se este período devido à maior estabilidade da economia brasileira após o Plano Real, já que dados anteriores poderiam conter distorções. Foram efetuadas as seguintes exclusões:

a) ações que não apresentavam cotações mensais consecutivas para o período de 12 meses anteriores ou 12 meses posteriores ao de formação das carteiras. Os meses anteriores seriam necessários para o cálculo do fator momento, e os posteriores para o cálculo dos retornos das ações. Os retornos das ações, por sua vez, seriam utilizados para a obtenção dos prêmios pelos fatores de risco;

b) ações sem valor de mercado em 31 de dezembro e 30 de junho, com tolerância de 23 dias;

c) ações de empresas que não possuíam Patrimônio Líquido positivo em dezembro;

d) empresas financeiras, devido a seu alto grau de endividamento, característico do setor. A exclusão decorre da influência que o endividamento tem sobre o índice $\mathrm{B} / \mathrm{M}$ e do fato do endividamento de empresas financeiras não ter o mesmo significado do endividamento de empresas não-financeiras; $e$

e) empresas com ações ON (ordinárias) e PN (preferenciais), caso a ação não tenha apresentado valor de mercado em junho, com tolerância de 23 dias, para qualquer uma das classes.

Diante do tratamento detalhado acima, nota-se que a amostra final desta pesquisa não está livre do viés de sobrevivência. Porém, esse efeito está diminuído pelo fato das carteiras serem reformuladas a cada 12 meses, e não mantidas iguais durante todo o período (de 1995 a 2007).

A amostra foi dividida em duas: 1) uma para estimar os betas e as sensibilidades aos fatores de risco a partir de regressões temporais (primeiro passo); e 2) outra para testar os modelos propriamente ditos, por meio de regres- 
sões cross-section dos prêmios de risco de cada uma das carteiras, regredidos contra as estimativas dos betas e as estimativas dos fatores, calculados no primeiro passo. A primeira é denominada within-sample e compreende o período de julho/1995 a dezembro/2001. A segunda, chamada de out-ofsample, compreende o período de janeiro/2002 a junho/2007. O ponto de corte em 2001 pautou-se, principalmente, no balanceamento do número de observações entre as amostras. Além disso, para dar maior robustez às análises e evitar possíveis problemas causados pela seleção amostral, utilizou-se outros quatro períodos de within-sample e out-of-sample aumentando (diminuindo) o período deles de seis em seis meses.

\subsection{Preparação das Carteiras}

Em junho de cada ano t, de 1995 a 2006, todas as ações da amostra foram classificadas de acordo com o índice $\mathrm{B} / \mathrm{M}$ das empresas que a representam. $\mathrm{O}$ índice foi calculado conforme a equação 4:

$$
\text { (4) } B / M_{i, t}=\frac{V C_{P L, d e z(t-1)}}{V M_{P L, d e z(t-1)}}
$$

Em que, $B / M_{i, t}=$ índice $B / M$, calculado com dados de dezembro de $t-1$, utilizado para a formação das carteiras no ano $t ; C_{P L \text {,dez(t-1) }}=$ valor contábil do patrimônio líquido em 31 de dezembro do ano $t-1 ; V M_{P L, d e z(t-1)}=$ valor de mercado do patrimônio líquido em 31 de dezembro do ano t-1. Para evitar distorções do índice B/M das empresas, o valor de mercado do patrimônio líquido foi obtido através da soma dos valores de mercado das ações ON e PN.

A amostra foi então separada de acordo com o valor do índice $\mathrm{B} / \mathrm{M}$ em três grupos: 30\% inferiores (Low), 40\% médios (Medium) e 30\% superiores (High). Utilizou-se o mês de junho pois, conforme Fama e French (1992), os valores de mercados nesse mês já refletem todas as informações contábeis anteriores.

Também em junho de cada ano $t$, todas as ações foram ordenadas de acordo com o valor de mercado de junho das empresas representadas, conforme descrito na equação 5 :

$$
\text { (5) } V M_{i, t}=\sum P_{y, i, t} \times N_{y, i, t}
$$


Onde, $V M_{i, t}=$ valor de mercado da empresa $i$ no momento $t ; P_{y, i, t}=$ preço da ação do tipo $y$, da empresa $i$ no momento $t ; N_{y, i, t}=$ número de ações do tipo $y$, da empresa $i$, no momento $t$. O valor da mediana foi utilizado para dividir a amostra em dois grupos: B (Big) e S (Small), contendo as empresas de maior e de menor valor de mercado, respectivamente.

Nesse mesmo mês, todas as ações foram ordenadas de acordo com o desempenho acumulado do retorno no período $t-2$ e $t-12$. Dessa forma, foi considerada a estratégia de momento de um ano de Jegadeesh e Titman (1993) e Carhart (1997). Na seqüência, a amostra foi separada pelo valor mediano em dois grupos: Los (Losers) e Win (Winners), contendo as empresas de piores e de melhores retornos históricos acumulados, respectivamente. Em junho de cada ano $t$, após as três ordenações anteriores, construíramse doze carteiras, decorrentes da interseção dos diversos grupos criados. As doze carteiras estão descritas no Quadro 1.

De julho do ano $t$ a junho de $t+1$, calculou-se o retorno mensal de cada ação, conforme demonstrado na equação 6:

$$
\text { (6) } R_{i, t}=\frac{\operatorname{Div}_{i, t}}{P_{i, t-1}}+\frac{P_{i, t}-P_{i, t-1}}{P_{i, t-1}}
$$

Onde, $R_{i, t}=$ retorno da ação $i$ para o mês $t ; \operatorname{Div}_{i, t}=$ dividendos da ação $i$ distribuídos durante o mês $t ; P_{i, t-1}=$ valor da ação $i$ ao final do mês $t-1 ; P_{i, t}=$ valor da ação $i$ ao final do mês $t$.

\begin{tabular}{|l|l|}
\hline Carteira & \multicolumn{1}{|c|}{ Descrição } \\
\hline H/B/Los & $\begin{array}{l}\text { (High, Big and Loser) - ações com alto índice B/M, alto valor de mercado e baixo } \\
\text { desempenho passado. }\end{array}$ \\
\hline H/B/Win & $\begin{array}{l}\text { (High, Big and Winner) - ações com alto índice B/M, alto valor de mercado e alto } \\
\text { desempenho passado. }\end{array}$ \\
\hline H/S/Los & $\begin{array}{l}\text { (High, Small and Loser) - ações com alto índice B/M, baixo valor de mercado e } \\
\text { baixo desempenho passado. }\end{array}$ \\
\hline H/S/Win & $\begin{array}{l}\text { (High, Small and Winner) - ações com alto índice B/M, baixo valor de mercado e } \\
\text { alto desempenho passado. }\end{array}$ \\
\hline L/B/Los & $\begin{array}{l}\text { (Low, Big and Loser) - ações com baixo índice B/M, alto valor de mercado e baixo } \\
\text { desempenho passado. }\end{array}$ \\
\hline L/B/Win & $\begin{array}{l}\text { (Low, Big and Winner) - ações com baixo índice B/M, alto valor de mercado e alto } \\
\text { desempenho passado. }\end{array}$ \\
\hline
\end{tabular}

Quadro 1: Descrição das 12 carteiras formadas a partir do tamanho (size), book-to-market e momento.

Fonte: Elaborado pelos autores. 


\begin{tabular}{|l|l|}
\hline Carteira & \multicolumn{1}{|c|}{ Descrição } \\
\hline L/S/Los & $\begin{array}{l}\text { (Low, Small and Loser) - ações com baixo índice B/M, baixo valor de mercado e } \\
\text { baixo desempenho passado. }\end{array}$ \\
\hline L/S/Win & $\begin{array}{l}\text { (Low, Small and Winner) - ações com baixo índice B/M, baixo valor de mercado e } \\
\text { alto desempenho passado. }\end{array}$ \\
\hline M/B/Los & $\begin{array}{l}\text { (Medium, Big and Loser) - ações com médio índice B/M, alto valor de mercado e } \\
\text { baixo desempenho passado. }\end{array}$ \\
\hline M/B/Win & $\begin{array}{l}\text { (Medium, Big and Winner) - ações com médio índice B/M, alto valor de mercado e } \\
\text { alto desempenho passado. }\end{array}$ \\
\hline M/S/Los & $\begin{array}{l}\text { (Medium, Small and Loser) - ações com médio índice B/M, baixo valor de } \\
\text { mercado e baixo desempenho passado. }\end{array}$ \\
\hline M/S/Win & $\begin{array}{l}\text { (Medium, Small and Winner) - ações com médio índice B/M, baixo valor de } \\
\text { mercado e alto desempenho passado. }\end{array}$ \\
\hline
\end{tabular}

Quadro 1: Descrição das 12 carteiras formadas a partir do tamanho (size), book-to-market e momento.

Fonte: Elaborado pelos autores.

\subsection{Regressões Temporais ( $1^{\text {a }}$ etapa do teste)}

O retorno mensal das carteiras foi obtido utilizando a equação 7:

$$
\text { (7) } R_{p, t}=\sum_{i=1}^{n} \frac{V M_{i t}}{V M_{p t}}\left(R_{i, t}\right)
$$

Em que, $R_{p, t}=$ retorno da carteira $p$ no mês $t ; R_{i, t}=$ retorno da ação $i$, pertencente à carteira $p$, no mês $t ; V M_{i, t}=$ valor de mercado da ação $i$, ao fim do mês $t$; $V M_{p, t}=$ valor de mercado da carteira $p$, ao fim do mês $t$.

$\mathrm{O}$ excesso de retorno mensal dessas doze carteiras em relação à taxa livre de risco foi a variável dependente da regressão linear temporal. Adotou-se o retorno mensal da caderneta de poupança como uma aproximação para a taxa livre de risco, conforme sugerido por Barros, Famá e Silveira (2003). As doze carteiras foram reformuladas em junho de cada ano, através da repetição das etapas acima.

Os prêmios pelo fator de risco mercado foram obtidos utilizando a equação 8: 
(8) Prêmio de Mercado $\mathrm{t}=\left[\sum_{\mathrm{i}=1}^{\mathrm{n}} \frac{V M_{i, t}}{V M_{M, t}}\left(R_{i, t}\right)\right]-R f_{t}$

Onde, $R_{i, t}=$ retorno da ação $i$, pertencente à carteira de mercado, no mês $t ; V M_{i, t}=$ valor de mercado da ação $i$, ao final do mês $t ; V M_{M, t}=$ valor de mercado da carteira de mercado, considerando todas as ações da amostra, no mês $t ; R f_{t}=$ retorno do ativo livre de risco, no mês $t$. $(\mathrm{SMB})$ :

A equação 9 foi utilizada para cálculo dos prêmios pelo fator tamanho

$$
\text { (9) } S M B_{t}=\overline{R_{S, t}}-\overline{R_{B, t}}
$$

Em que, $S M B_{t}=$ prêmio pelo fator tamanho, no mês $t ; R_{S, t}=$ retorno médio mensal das seis carteiras $\mathrm{S} ; R_{B, t}=$ retorno médio mensal das seis carteiras B.

Os prêmios pelo fator índice $\mathrm{B} / \mathrm{M}$ (HML) foram calculados conforme a equação 10:

$$
\text { (10) } H M L_{t}=\overline{R_{H, t}}-\overline{R_{L, t}}
$$

Onde, $H M L_{t}=$ prêmio mensal pelo fator de risco $\mathrm{B} / \mathrm{M} ; R_{H, t}=$ retorno médio mensal das quatro carteiras $\mathrm{H} ; R L_{, t}=$ retorno médio mensal das quatro carteiras L.

Por sua vez, os prêmios pelo fator momento (WinMLos) foram obtidos utilizando a equação 11:

$$
\text { (11) WinMLoSt }=\overline{R_{W I N, t}}-\overline{R_{L O S, t}}
$$

Em que, WinMLos ${ }_{t}=$ prêmio mensal pelo fator de risco momento; $R_{\text {WIN,t }}$ $=$ retorno médio mensal das seis carteiras Win, dado por: $R_{L O S, t}=$ retorno médio mensal das seis carteiras Los.

Esses quatro prêmios mensais foram utilizados como variáveis explicativas das regressões temporais para obtenção dos betas dos fatores de risco. 


\subsection{Regressões Cross-Section ( $2^{\mathrm{a}}$ etapa do teste)}

Nessa etapa, os betas dos fatores dos modelos estimados nas regressões em séries temporais (a partir da amostra within-sample) são usados como variáveis explicativas dos retornos cross-section (na amostra out-of-sample). As variáveis dependentes das regressões são os excessos de retorno mensal das carteiras em relação à taxa livre de risco.

Acrescenta-se que os erros-padrão dos coeficientes das regressões crosssection não são obtidos pela metodologia convencional de Mínimos Quadrados Ordinários (MQO). Devido à incapacidade de identificar o "verdadeiro beta" (ou verdadeiras sensibilidades) o método MQO pode produzir estimativas totalmente enviesadas. No caso do CAPM, o beta obtido através da regressão em séries temporais é uma estimativa do "verdadeiro beta". Mesmo que houvesse um beta verdadeiro e estável, tudo o que existe é uma estimativa, e essa estimativa, ainda que possa ser não enviesada, está sujeita a erro de amostragem. Dessa forma, qualquer erro na estimativa do beta fará com que o coeficiente beta na regressão cross-section seja enviesado para baixo e o intercepto seja enviesado para cima (ELTON et al., 2004, p. 300).

Além do mais, à medida que o verdadeiro valor do beta está positivamente correlacionado com a variância do resíduo, esta acabará funcionando como proxy do verdadeiro beta e o retorno ficará positivamente correlacionado com o risco residual (ELTON et al., 2004, p. 300). Catalogados esses possíveis problemas para testes dos modelos de retornos esperados, Fama e Macbeth (1973) indicaram duas contribuições para diminuir o erro amostral e a correlação (com qualquer defasagem) entre os resíduos da equação (risco residual): 1) medir betas de carteiras e não de títulos individuais; e 2) estimar os betas a partir de $n$ regressões cross-sections mensais e usar o valor médio dos parâmetros como coeficiente da equação cross-section de todo período (equação geral). Essa última indicação ficou conhecida como técnica de Fama e Macbeth (1973) para cômputo dos erros-padrão.

Fama e Macbeth (1973) formaram 20 carteiras de títulos para estimação de betas numa regressão de primeiro passo (com dados em séries temporais), e, a seguir, fizeram $n$ regressões de segundo passo (com dados em cross-section) para cada mês do período. Ao se estimar a equação para cada mês, é possível examinar como os parâmetros variam no tempo, e reduzir possível correlação serial entre o risco residual e o "verdadeiro beta". A significância estatística de cada parâmetro (beta e sensibilidades aos fatores 
de risco) da equação geral pode ser determinada calculando o desvio-padrão da média e testando se a média está a um número significantemente alto de desvios-padrão distante de zero.

Algumas hipóteses podem ser formuladas para validação dos modelos CAPM, 3-fatores e 4-fatores. Nessa etapa espera-se que: 1) as equações apresentem interceptos iguais a zero, ou seja, não se espera retorno adicional em cada modelo; 2) nenhuma variável adicional seja relevante na explicação das variações dos retornos das carteiras; e 3) Em relação ao CAPM, quanto maior o risco (medido pelo beta) maior deve ser o retorno, numa relação linear.

\section{Análise dos Dados e Resultados}

\subsection{Sensibilidade dos Excessos de Retorno aos Fatores de Risco}

A Tabela 1 apresenta os resultados das regressões com as séries temporais considerando todo o período amostral. Nessa tabela demonstram-se as sensibilidades dos excessos de retorno mensal aos quatro fatores incluídos no modelo.

O excesso de retorno médio mensal variou de $0,058 \%$ a 2,736\%. Málaga e Securato (2004) e Mussa, Santos e Famá (2007) chegaram a prêmios mensais oscilando de $0,06 \%$ a $1,87 \%$ e de $0,04 \%$ a $2,72 \%$, respectivamente. Fama e French (1993) constataram que os prêmios mensais variam de 0,32\% a $1,05 \%$ para o mercado norte-americano. Vale notar que não se pode confirmar que as empresas de menor porte apresentam maior retorno por representarem maior risco, dado que muitas das carteiras B apresentam retornos médios superiores ao de carteiras S. Percebe-se também que as carteiras Los, em muitos casos, apresentam retornos médios superiores aos de carteiras Win. Esse fato não permite confirmar a suposição de Jegadeesh e Titman (1993), de que a estratégia de comprar ações de empresas que tiveram alto desempenho no último ano (Win) leva a um retorno superior em comparação com a estratégia de se optar por ações de empresas que apresentaram baixo desempenho no último ano (Los).

Os coeficientes do fator beta de mercado $(b)$ mostraram-se maiores e mais próximos de 1 quando foram adicionados os demais fatores do modelo 4-fatores, em comparação ao CAPM. O mesmo foi verificado por Fama e 
French (1993), Málaga e Securato (2004) e Mussa, Santos e Famá (2007). Segundo Fama e French (1993) este fato estaria relacionado à correlação dos demais fatores com o fator mercado.

Os coeficientes do fator tamanho $(s)$ mostraram-se maiores nas carteiras $\mathrm{S}$, indicando que este fator explica melhor as carteiras formadas por empresas de pequeno porte. Já os coeficientes do fator índice B/M $(h)$ apresentaram valores crescentes à medida que se passou das carteiras $L$ para as carteiras $\mathrm{H}$, parecendo então explicar melhor as carteiras formadas por ações de alto índice B/M. Por fim, os valores dos coeficientes do fator momento $w$ mostraram-se mais expressivos em carteiras Los, formadas por ações que tiveram um baixo retorno acumulado nos últimos 12 meses.

Tabela 1: Prêmio pelo risco, beta do CAPM e sensibilidades dos quatro fatores para as 12 carteiras formadas a partir do tamanho (size), do book-to-market e do momento.

\begin{tabular}{|l|c|c|c|c|c|c|}
\hline Carteira & $\begin{array}{c}\text { Excesso } \\
\text { Retorno }\end{array}$ & $\begin{array}{c}\text { Beta } \\
\text { (CAPM) }\end{array}$ & $\boldsymbol{b}$ & $\boldsymbol{s}$ & $\boldsymbol{h}$ & $\boldsymbol{w}$ \\
\hline HBLOS & 1,867 & 1,108 & 0,932 & 0,069 & 0,861 & $-0,939$ \\
\hline HBWIN & 1,726 & 1,066 & 1,061 & 0,108 & 0,823 & 0,465 \\
\hline HSLOS & 2,736 & 0,574 & 0,900 & 1,075 & 0,677 & $-0,417$ \\
\hline HSWIN & 1,592 & 0,598 & 0,971 & 0,990 & 0,644 & 0,312 \\
\hline LBLOS & 0,621 & 0,979 & 0,922 & $-0,077$ & $-0,226$ & $-0,497$ \\
\hline LBWIN & 1,082 & 0,815 & 0,867 & 0,026 & $-0,174$ & 0,184 \\
\hline LSLOS & 1,335 & 0,646 & 1,065 & 1,049 & $-0,288$ & $-0,549$ \\
\hline LSWIN & 0,058 & 0,418 & 1,010 & 1,244 & $-0,307$ & 0,282 \\
\hline MBLOS & 1,788 & 0,985 & 0,907 & $-0,028$ & 0,014 & $-0,603$ \\
\hline MBWIN & 2,588 & 0,993 & 1,080 & 0,084 & 0,033 & 0,519 \\
\hline MSLOS & 1,567 & 0,710 & 1,043 & 1,093 & 0,291 & $-0,866$ \\
\hline MSWIN & 2,572 & 0,471 & 0,780 & 0,730 & 0,311 & 0,367 \\
\hline
\end{tabular}

Fonte: Elaborada pelos autores.

Nota: Os cálculos nessa tabela são procedidos usando todo período da amostra: julho de 1995 a junho de 2007. O excesso de retorno (\% média mensal) é a média mensal do prêmio pelo risco na amostra. O beta do CAPM das carteiras é obtido pela regressão em séries temporais de seus excessos de retornos mensais sobre o excesso mensal de retorno do mercado. A carteira de mercado é formada a partir das ações da amostra, que são ponderadas pelo valor de mercado, e da taxa livre de risco, que é o retorno da Caderneta de Poupança. As sensibilidades aos quatro fatores são obtidas através das regressões de séries temporais: a variável dependente representa os prêmios mensais pelo risco das carteiras;

$R_{M t}-R_{F t}$ significa os excessos mensais de retornos do mercado; SML e HML são os retornos dos fatores tamanhos ("small menos big") e book-to-market ("high menos low"); WinMLos traduz o fator momento ("winners menos losers"). 


\subsection{Testes dos Modelos}

A Tabela 2 traz os resultados das regressões cross-section dos prêmios mensais pelo risco das carteiras $\left(R_{i}-R_{f}\right)$, calculados out-of-sample, sobre o beta do CAPM e as sensibilidades dos quatro fatores para as 12 carteiras formadas a partir das variáveis tamanho, índice $\mathrm{B} / \mathrm{M}$ e momento. As entradas da tabela são os valores dos coeficientes estimados e entre parênteses o $t$ estatístico. Os valores dos interceptos dos três modelos (CAPM, 3-fatores e 4fatores) foram altamente significativos, indicando que esses modelos não se mostram suficientes na explicação do excesso de retorno das carteiras.

Nos testes com os interceptos, o beta não se mostrou significativo no modelo CAPM e o $R^{2} A d j$. foi bastante baixo $(0,030)$. No modelo 3 -fatores, o beta de mercado foi o único fator significativo e o $R^{2} A d j$. se elevou para 0,596 . No modelo 4-fatores, os fatores mercado e índice B/M mostraram-se significativos, com o $R^{2}$ Adj. de 0,684.

Com a exclusão dos interceptos, o beta de mercado passou a ser significativo no modelo CAPM, mas o $R^{2} A d j$. manteve-se insignificante. Já nos modelos 3-fatores e 4-fatores, os fatores beta de mercado e índice $\mathrm{B} / \mathrm{M}$ mostraram-se significativos. Os fatores tamanho e momento continuaram insignificantes nos modelos em que foram incluídos. Novamente o maior $R^{2} A d j$. foi obtido no modelo 4-fatores, seguido pelo modelo 3-fatores e CAPM.

Tabela 2: Resultados das regressões cross-section dos prêmios mensais pelo risco das carteiras $\left(R_{j}-R_{F}\right)$, calculados out-of-sample sobre o beta do CAPM.

\begin{tabular}{|c|c|c|c|c|c|c|c|}
\hline Modelo & Interceptora & $\begin{array}{c}\text { Beta } \\
\text { (CAPM) }\end{array}$ & b & $s$ & $\boldsymbol{h}$ & $\mathbf{w}$ & $\begin{array}{r}\text { R2 } \\
\text { Adj. }\end{array}$ \\
\hline $\begin{array}{l}\text { CAPM + } \\
\text { Intercepto }\end{array}$ & $\begin{array}{l}3,39 \\
(4,14) * *\end{array}$ & $\begin{array}{l}-1,36 \\
(-1,28) \\
\end{array}$ & & & & & 0,030 \\
\hline $\begin{array}{l}\text { 3-Fatores + } \\
\text { Intercepto }\end{array}$ & $\begin{array}{l}5,99 \\
(4,50) \quad * * \\
\end{array}$ & & $\begin{array}{l}-4,38 \\
(-3,06) * *\end{array}$ & $\begin{array}{l}0,57 \\
(1,21) \\
\end{array}$ & $\begin{array}{l}1,31 \\
(2,17) \\
\end{array}$ & & $0,596 *$ \\
\hline $\begin{array}{l}\text { 4-Fatores + } \\
\text { Intercepto }\end{array}$ & $\begin{array}{l}6,18 \\
(4,39) \quad * *\end{array}$ & & $\begin{array}{l}-4,45 \\
(-3,05) * *\end{array}$ & $\begin{array}{l}0,45 \\
(0,93) \\
\end{array}$ & $\begin{array}{l}1,32 \\
(2,20)^{*}\end{array}$ & $\begin{array}{l}0,81 \\
(1,97)\end{array}$ & $0,684^{*}$ \\
\hline CAPM & & $\begin{array}{l}2,73 \\
(3,07)^{* *}\end{array}$ & & & & & $-0,069$ \\
\hline 3-Fatores & & & $\begin{array}{l}1,77 \\
(2,22)^{*}\end{array}$ & $\begin{array}{l}0,56 \\
(1,18)\end{array}$ & $\begin{array}{l}1,60 \\
(2,71)^{* *}\end{array}$ & & $0,471 *$ \\
\hline 4-Fatores & & & $\begin{array}{l}1,88 \\
(2,38)^{*}\end{array}$ & $\begin{array}{l}0,46 \\
(0,95)\end{array}$ & $\begin{array}{l}1,62 \\
(2,76) * *\end{array}$ & $\begin{array}{l}0,55 \\
(1,44)\end{array}$ & $0,541 *$ \\
\hline
\end{tabular}

Fonte: Elaborada pelos autores. 
Nota: Nessa tabela é simulada a robustez dos resultados em diferentes períodos da amostra. A data na coluna within-sample representa o período de cálculo dos betas do CAPM e as sensibilidades dos quatro fatores para as 12 carteiras formadas. O período out-of-sample compreende o mês imediatamente posterior até junho de 2007. Nessas regressões as variáveis dependentes são as médias mensais dos prêmios pelo risco das carteiras calculados out-of-sample, e as variáveis explicativas são os betas do $\mathrm{CAPM}$ e as sensibilidades dos quatro fatores das carteiras calculados within-sample, a partir das regressões em séries temporais. Na tabela são reportados os coeficientes das regressões e entre parênteses o valor do t-estatístico. ${ }^{*}$ indica que o coeficiente é significativo a $1 \%$; $e^{*}$ significativo a $5 \%$. Os erros-padrão para cômputo do t-estatístico foram calculados conforme a técnica de Fama e Macbeth (1973).

Para minimizar os possíveis prejuízos causados pela definição arbitrária dos períodos de within-sample e out-of-sample e dar maior robustez aos procedimentos empíricos, os testes foram efetuados considerando outros quatro períodos de separação da amostra. Os resultados são apresentados na Tabela 3.

Em todas as situações, os interceptos dos três modelos mostraram-se altamente significativos, corroborando que os modelos parecem não ser suficientes na explicação dos retornos das carteiras. Também não se observaram alterações significativas no comportamento dos fatores de risco. Os fatores tamanho e momento não foram significativos em nenhum dos modelos, com ou sem os interceptos. O fator beta no CAPM mostrou-se significativo também somente quando os interceptos foram excluídos. O beta de mercado e o índice $\mathrm{B} / \mathrm{M}$ mantiveram-se como os fatores que mais parecem explicar as variações dos retornos cross-section, mas, ainda assim, a significância destes fatores oscilou à medida que se modificaram os períodos de within-sample e out-of-sample.

Finalmente, para se testar a terceira hipótese da pesquisa, ou seja, a linearidade do modelo CAPM, foram realizadas regressões cross-section dos prêmios mensais pelo risco das carteiras $\left(R_{j}-R_{F}\right)$, calculados em diferentes out-of-sample, sobre o beta do CAPM e o beta do CAPM ao quadrado, para as 12 carteiras formadas a partir dos fatores tamanho, índice $\mathrm{B} / \mathrm{M}$ e momento. Os resultados são apresentados na Tabela 4 e agregam evidências no sentido de rejeição do CAPM, uma vez que os interceptos foram altamente significativos e, quando excluídos, observou-se que betas ao quadrado mostraram-se também altamente significativos em todos os períodos de out-of-sample considerados. 
Tabela 3: Regressões cross-section dos prêmios mensais pelo risco das carteiras $\left(R_{j}-R_{F}\right)$, calculados em diferentes out-of-sample, sobre o beta do CAPM e o beta do CAPM ao quadrado para as 12 carteiras formadas a partir do tamanho, book-to-market e momento.

\begin{tabular}{|c|c|c|c|c|c|c|c|}
\hline $\begin{array}{l}\text { Within- } \\
\text { sample }\end{array}$ & Modelo & Interceptora & $\begin{array}{c}\text { Beta } \\
\text { (CAPM) }\end{array}$ & $\boldsymbol{b}$ & $s$ & $\boldsymbol{h}$ & $\mathbf{w}$ \\
\hline \multirow{6}{*}{$\begin{array}{c}\text { julho } \\
\text { de } 1995 \\
\text { a } \\
\text { dezembro } \\
\text { de } 2000\end{array}$} & $\begin{array}{l}\text { CAPM + } \\
\text { Intercepto }\end{array}$ & $\begin{array}{l}2,91 \\
(3,97) * *\end{array}$ & $\begin{array}{l}-1,18 \\
(-1,29)\end{array}$ & & & & \\
\hline & $\begin{array}{l}\text { 3-Fatores + } \\
\text { Intercepto }\end{array}$ & $\begin{array}{l}4,47 \\
(3,26) * *\end{array}$ & & $\begin{array}{l}-3,13 \\
(-2,29)^{*}\end{array}$ & $\begin{array}{l}0,51 \\
(1,19)\end{array}$ & $\begin{array}{l}1,16 \\
(2,04) *\end{array}$ & \\
\hline & $\begin{array}{l}\text { 4-Fatores + } \\
\text { Intercepto }\end{array}$ & $\begin{array}{l}4,61 \\
(3,23)^{* *}\end{array}$ & & \begin{tabular}{|l|}
$-3,17$ \\
$(-2,29) *$ \\
\end{tabular} & $\begin{array}{l}0,42 \\
(0,95) \\
\end{array}$ & $\begin{array}{l}1,16 \\
(2,04) *\end{array}$ & $\begin{array}{l}0,64 \\
(1,82)\end{array}$ \\
\hline & CAPM & & $\begin{array}{l}2,36 \\
(2,68) * *\end{array}$ & & & & \\
\hline & 3-Fatores & & & $\begin{array}{l}1,50 \\
(1,88)\end{array}$ & $\begin{array}{l}0,54 \\
(1,27)\end{array}$ & $\begin{array}{l}1,53 \\
(2,84) * *\end{array}$ & \\
\hline & 4-Fatores & & & $\begin{array}{l}1,58 \\
(1,99) \\
\end{array}$ & $\begin{array}{l}0,47 \\
(1,07) \\
\end{array}$ & $\begin{array}{l}1,54 \\
(2,87)^{* *}\end{array}$ & $\begin{array}{l}0,47 \\
(1,42)\end{array}$ \\
\hline \multirow{6}{*}{$\begin{array}{c}\text { julho de } \\
1995 \\
\text { a } \\
\text { junho de } \\
2001\end{array}$} & $\begin{array}{l}\text { CAPM + } \\
\text { Intercepto }\end{array}$ & $\begin{array}{l}3,01 \\
(3,83) * *\end{array}$ & $\begin{array}{l}-1,12 \\
(-1,16)\end{array}$ & & & & \\
\hline & $\begin{array}{l}\text { 3-Fatores + } \\
\text { Intercepto }\end{array}$ & $\begin{array}{l}4,45 \\
(3,27)^{* *}\end{array}$ & & $\begin{array}{l}-3,01 \\
(-2,29)^{*}\end{array}$ & $\begin{array}{l}0,55 \\
(1,25)\end{array}$ & $\begin{array}{l}1,39 \\
(2,51) *\end{array}$ & \\
\hline & $\begin{array}{l}\text { 4-Fatores + } \\
\text { Intercepto }\end{array}$ & $\begin{array}{l}4,59 \\
(3,25) * *\end{array}$ & & $\begin{array}{l}-3,05 \\
(-2,29)^{*}\end{array}$ & $\begin{array}{l}0,45 \\
(0,99)\end{array}$ & $\begin{array}{l}1,41 \\
(2,54) *\end{array}$ & $\begin{array}{l}0,70 \\
(1,82)\end{array}$ \\
\hline & CAPM & & $\begin{array}{l}2,54 \\
(2,85) * *\end{array}$ & & & & \\
\hline & 3-Fatores & & & $\begin{array}{l}1,60 \\
(1,99)\end{array}$ & $\begin{array}{l}0,55 \\
(1,25)\end{array}$ & $\begin{array}{l}1,66 \\
(3,04) * *\end{array}$ & \\
\hline & 4-Fatores & & & $\begin{array}{l}1,70 \\
(2,13)^{*}\end{array}$ & $\begin{array}{l}0,46 \\
(1,02) \\
\end{array}$ & $\begin{array}{l}1,68 \\
(3,10) * *\end{array}$ & $\begin{array}{l}0,53 \\
(1,48)\end{array}$ \\
\hline \multirow{6}{*}{$\begin{array}{c}\text { julho de } \\
1995 \\
\text { a } \\
\text { junho de } \\
2002\end{array}$} & $\begin{array}{l}\text { CAPM + } \\
\text { Intercepto }\end{array}$ & $\begin{array}{l}3,95 \\
(4,59) * *\end{array}$ & $\begin{array}{l}-1,58 \\
(-1,41)\end{array}$ & & & & \\
\hline & $\begin{array}{l}\text { 3-Fatores + } \\
\text { Intercepto }\end{array}$ & $\begin{array}{l}6,11 \\
(4,10) * *\end{array}$ & & $\begin{array}{l}-4,02 \\
(-2,52)^{*}\end{array}$ & $\begin{array}{l}0,62 \\
(1,23)\end{array}$ & $\begin{array}{l}0,97 \\
(1,55)\end{array}$ & \\
\hline & $\begin{array}{l}\text { 4-Fatores + } \\
\text { Intercepto }\end{array}$ & $\begin{array}{l}6,11 \\
(4,10)^{* *}\end{array}$ & & $\begin{array}{l}-3,92 \\
(-2,56)^{*}\end{array}$ & $\begin{array}{l}0,53 \\
(1,04)\end{array}$ & $\begin{array}{l}0,98 \\
(1,57) \\
\end{array}$ & $\begin{array}{l}0,73 \\
(1,58)\end{array}$ \\
\hline & CAPM & & $\begin{array}{l}3,18 \\
(3,45)^{* *}\end{array}$ & & & & \\
\hline & 3-Fatores & & & $\begin{array}{l}2,22 \\
(2,72) * * \\
\end{array}$ & $\begin{array}{l}0,63 \\
(1,25)\end{array}$ & $\begin{array}{l}1,28 \\
(2,09) *\end{array}$ & \\
\hline & 4-Fatores & & & $\begin{array}{l}2,32 \\
(2,86) * *\end{array}$ & $\begin{array}{l}0,54 \\
(1,06)\end{array}$ & $\begin{array}{l}1,28 \\
(2,10)^{*}\end{array}$ & $\begin{array}{l}0,46 \\
(1,09)\end{array}$ \\
\hline
\end{tabular}

Fonte: Elaborada pelos autores. 
Tabela 3: Regressões cross-section dos prêmios mensais pelo risco das carteiras $\left(R_{j}-R_{F}\right)$, calculados em diferentes out-of-sample, sobre o beta do CAPM e o beta do CAPM ao quadrado para as 12 carteiras formadas a partir do tamanho, book-to-market e momento.

\begin{tabular}{|c|c|c|c|c|c|c|c|}
\hline $\begin{array}{l}\text { Within- } \\
\text { sample }\end{array}$ & Modelo & Interceptora & $\begin{array}{c}\text { Beta } \\
\text { (CAPM) }\end{array}$ & b & $s$ & $\boldsymbol{h}$ & $\mathbf{w}$ \\
\hline \multirow{6}{*}{$\begin{array}{c}\text { julho de } \\
1995 \\
\text { a } \\
\text { dezembro } \\
\text { de } 2002\end{array}$} & $\begin{array}{l}\text { CAPM + } \\
\text { Intercepto }\end{array}$ & $\begin{array}{l}3,94 \\
(4,24) * *\end{array}$ & $\begin{array}{l}-1,22 \\
(-1,08)\end{array}$ & & & & \\
\hline & $\begin{array}{l}\text { 3-Fatores + } \\
\text { Intercepto }\end{array}$ & $\begin{array}{l}5,44 \\
(3,41) * *\end{array}$ & & $\begin{array}{l}-3,01 \\
(-1,83)\end{array}$ & $\begin{array}{l}0,54 \\
(1,04)\end{array}$ & $\begin{array}{l}0,96 \\
(1,47)\end{array}$ & \\
\hline & $\begin{array}{l}\text { 4-Fatores + } \\
\text { Intercepto }\end{array}$ & $\begin{array}{l}5,40 \\
(3,47)^{* *}\end{array}$ & & $\begin{array}{l}-2,90 \\
(-1,89)\end{array}$ & $\begin{array}{l}0,49 \\
(0,94)\end{array}$ & $\begin{array}{l}0,98 \\
(1,52)\end{array}$ & $\begin{array}{l}0,50 \\
(0,99)\end{array}$ \\
\hline & CAPM & & $\begin{array}{l}3,49 \\
(3,77)^{* *}\end{array}$ & & & & \\
\hline & 3-Fatores & & & $\begin{array}{l}2,53 \\
(3,17)^{* *}\end{array}$ & $\begin{array}{l}0,53 \\
(1,03)\end{array}$ & $\begin{array}{l}1,22 \\
(1,91)\end{array}$ & \\
\hline & 4-Fatores & & & $\begin{array}{l}2,60 \\
(3,30)^{* *}\end{array}$ & $\begin{array}{l}0,47 \\
(0,91)\end{array}$ & $\begin{array}{l}1,24 \\
(1,96)\end{array}$ & $\begin{array}{l}0,30 \\
(0,64)\end{array}$ \\
\hline
\end{tabular}

Fonte: Elaborada pelos autores.

Nota: O objetivo dos valores apresentados nessa tabela é testar a hipótese de linearidade do modelo CAPM. Nesse sentido, é simulado, em diferentes períodos da amostra, o modelo CAPM, com a inclusão do beta ao quadrado e uma constante. A data na coluna within-sample representa o período de cálculo dos betas do CAPM e dos betas ao quadrado para as 12 carteiras formadas a partir do tamanho, do book-tomarket e do momento. O período out-of-sample compreende o mês imediatamente posterior até junho de 2007. Nessas regressões as variáveis dependentes são as médias mensais dos prêmios pelo risco das carteiras calculados out-of-sample e as variáveis explicativas são os betas do CAPM e os betas ao quadrado das carteiras calculadas within-sample, a partir das regressões em séries temporais. Na tabela são reportados os coeficientes das regressões e entre parênteses o valor do t-estatístico. ** indica que o coeficiente é significativo a $1 \%$; $e^{*}$ significativo a $5 \%$. Os erros-padrão para cômputo do t-estatístico foram calculados conforme a técnica de Fama e Macbeth (1973). 
Tabela 4: Regressões cross-section dos prêmios mensais pelo risco das carteiras $\left(R_{j}-R_{F}\right)$, calculados em diferentes out-of-sample, sobre o beta do CAPM ao quadrado para as 12 carteiras formadas a partir do tamanho, book-to-market e momento.

\begin{tabular}{|c|c|c|c|c|}
\hline Within-sample & Modelo & Interceptora & Beta^ 2 & Beta \\
\hline \multirow{2}{*}{$\begin{array}{c}\text { julho de } 1995 \\
\text { a } \\
\text { dezembro de } 2000\end{array}$} & $\begin{array}{l}\mathrm{CAPM} \wedge 2+ \\
\text { Intercepto }\end{array}$ & $\begin{array}{l}4,59 \\
(3,09) * *\end{array}$ & $\begin{array}{l}3,84 \\
(1,33) \\
\end{array}$ & $\begin{array}{l}-6,62 \\
(-1,63) \\
\end{array}$ \\
\hline & $\mathrm{CAPM}^{\wedge} 2$ & & $\begin{array}{l}-5,43 \\
(-3,60) * *\end{array}$ & $\begin{array}{l}7,17 \\
(3,82) * *\end{array}$ \\
\hline \multirow{2}{*}{$\begin{array}{c}\text { julho de } 1995 \\
\text { a } \\
\text { junho de } 2001\end{array}$} & $\begin{array}{l}\text { CAPM^ } 2+ \\
\text { Intercepto }\end{array}$ & $\begin{array}{l}4,85 \\
(3,05) * *\end{array}$ & $\begin{array}{l}4,13 \\
(1,35)\end{array}$ & $\begin{array}{l}-7,01 \\
(-1,60)\end{array}$ \\
\hline & $\mathrm{CAPM}^{\wedge} 22$ & & $\begin{array}{l}-5,60 \\
(-3,50) * *\end{array}$ & $\begin{array}{l}7,49 \\
(3,81) * *\end{array}$ \\
\hline \multirow{2}{*}{$\begin{array}{c}\text { julho de } 1995 \\
\text { a } \\
\text { dezembro de } 2001\end{array}$} & $\begin{array}{l}\mathrm{CAPM} \wedge 2+ \\
\text { Intercepto }\end{array}$ & $\begin{array}{l}5,27 \\
(2,79) * *\end{array}$ & $\begin{array}{l}4,06 \\
(1,12)\end{array}$ & $\begin{array}{l}-7,24 \\
(-1,37)\end{array}$ \\
\hline & $\mathrm{CAPM}^{\wedge} 2$ & & $\begin{array}{l}-6,33 \\
(-3,88) * *\end{array}$ & $\begin{array}{l}8,35 \\
(4,30) * *\end{array}$ \\
\hline $\begin{array}{c}\text { julho de } 1995 \\
\text { a } \\
\text { junho de } 2002\end{array}$ & $\begin{array}{l}\mathrm{CAPM}^{\wedge} 2+ \\
\text { Intercepto } \\
\mathrm{CAPM}^{\wedge} 2\end{array}$ & $\begin{array}{l}6,18 \\
(3,21) * *\end{array}$ & $\begin{array}{l}4,81 \\
(1,28) \\
-7,33 \\
(-4,23) * *\end{array}$ & $\begin{array}{l}-8,54 \\
(-1,58) \\
9,71 \\
(4,76) * *\end{array}$ \\
\hline $\begin{array}{c}\text { julho de } 1995 \\
\text { a } \\
\text { dezembro de } 2002\end{array}$ & $\begin{array}{l}\mathrm{CAPM}^{\wedge} 2+ \\
\text { Intercepto } \\
\mathrm{CAPM}^{\wedge} 2\end{array}$ & $\begin{array}{l}5,88 \\
(2,80) * *\end{array}$ & $\begin{array}{l}4,12 \\
(1,04) \\
-7,34 \\
(-4,00) * *\end{array}$ & $\begin{array}{l}-7,23 \\
(-1,26) \\
10,06 \\
(4,69) * *\end{array}$ \\
\hline
\end{tabular}

Fonte: Elaborada pelos autores.

Nota: O objetivo dos valores apresentados nessa tabela é testar a hipótese de linearidade do modelo CAPM. Nesse sentido, é simulado, em diferentes períodos da amostra, o modelo CAPM, com a inclusão do beta ao quadrado e uma constante. A data na coluna within-sample representa o período de cálculo dos betas do CAPM e dos betas ao quadrado para as 12 carteiras formadas a partir do tamanho, do book-tomarket e do momento. O período out-of-sample compreende o mês imediatamente posterior até junho de 2007. Nessas regressões as variáveis dependentes são as médias mensais dos prêmios pelo risco das carteiras calculados out-of-sample e as variáveis explicativas são os betas do CAPM e os betas ao quadrado das carteiras calculadas within-sample, a partir das regressões em séries temporais. Na tabela são reportados os coeficientes das regressões e entre parênteses o valor do t-estatístico. ** indica que o coeficiente é significativo a $1 \%$; e significativo a $5 \%$. Os erros-padrão para cômputo do t-estatístico foram calculados conforme a técnica de Fama e Macbeth (1973). 


\section{Considerações Finais}

O objetivo do presente artigo foi testar e comparar os modelos CAPM, 3 -fatores e 4-fatores, ao se aplicar uma metodologia de teste preditivo, utilizando regressões em duas etapas: séries temporais e cross-section. Adicionalmente foi testada a hipótese de linearidade do CAPM. A amostra foi constituída por todas as ações listadas na Bolsa de Valores do Estado de São Paulo - BOVESPA - de 1995 a 2007. Para dar maior robustez às análises, foram considerados diferentes períodos de within-sample e out-of-sample.

Os resultados indicaram que nenhum dos três modelos testados foram suficientes na explicação das variações dos retornos das ações do mercado brasileiro, devido à alta significância dos interceptos. Apesar disso, o modelo 4-fatores apresentou valores de $R^{2} \mathrm{Adj}$. superiores aos dos modelos 3 -fatores e CAPM. O fator mercado não se mostrou significativo no modelo CAPM. Já nos modelos 3-fatores e 4-fatores, somente as variáveis mercado e índice $\mathrm{B} /$ $\mathrm{M}$ foram significativas. Os fatores tamanho e momento não foram significativos em nenhum dos modelos e períodos testados. Esses resultados sugerem a utilização de um modelo de dois fatores (mercado e índice $\mathrm{B} / \mathrm{M}$ ) para a predição de retornos esperados no mercado acionário brasileiro. Além disso, foram encontradas evidências no sentido de rejeição da hipótese de linearidade do CAPM.

Faz-se oportuno destacar que os resultados do presente trabalho devem ser considerados com ressalvas, devido às limitações da pesquisa. Uma importante limitação é a quantidade relativamente pequena de ações disponíveis no mercado brasileiro para a formação das carteiras deste estudo. Esse fato pode acarretar a criação de um número pequeno e pouco diversificado de carteiras, levando a prejuízos na estimação de modelos APT e nos testes cross-section. Porém, ressalta-se que nada pôde ser feito pelos autores em relação a esta limitação, uma vez que ela é motivada por características inerentes ao mercado acionário brasileiro.

Por fim, destaca-se que foram notadas algumas diferenças importantes nos resultados deste trabalho, quando comparado aos estudos efetuados anteriormente no mercado acionário brasileiro. A principal delas refere-se à relevância dos fatores tamanho e momento, que foram significativos nas pesquisas de Málaga e Securato (2004) e Mussa, Santos e Famá (2007), mas não foram no presente trabalho. Esse fato foi possivelmente motivado pela diferença metodológica entre os estudos. Desta forma, agregam-se evidên- 
cias locais no sentido de corroborar que o tema é ainda incipiente e objeto de muita polêmica dentro do meio acadêmico. Os resultados encontrados talvez tenham despertado mais questionamentos do que conclusões, mas, sem dúvida, contribuirão para o desenvolvimento de pesquisas futuras, dentre as quais se pode citar a variação dos critérios utilizados na metodologia $e$ a busca por fatores de risco significativos, específicos de países emergentes ou do Brasil.

\title{
Models of expected returns on the brazilian market: Empirical tests using predictive methodology
}

\begin{abstract}
Predictive methodologies for test of the expected returns models are largely diffused on the international academic environment. However, these methods have not been used in Brazil in a systematic way. Generally, empirical studies proceeded with Brazilian stock market data are concentrated only in the first step of these methodologies. The purpose of this article was test and compare the models CAPM, 3 -factors and 4-factors using a predictive methodology, considering two steps temporal and cross-section regressions - with standard errors obtained by the techniques of Fama and Macbeth (1973). The results indicated the superiority of the 4-fators model as compared to the 3-fators model, and the superiority of the 3factors model as compared to the CAPM, but no one of the tested models were enough on the explanation of the Brazilian stock returns. Contrary to some empirical evidences, that do not use predictive methodology, the size and momentum effect seem do not exist on the Brazilian capital markets, but there are evidences of the value effect and the relevance of the market for explanation of expected returns. These finds rise some questions, mainly caused by the originality of the methodology on the local market and by the fact that this subject is still incipient and polemic on the Brazilian academic environment.
\end{abstract}

Key-words: CAPM. Size Effect. Momentum Effect. Value Effect. 


\section{REFERÊNCIAS}

BANZ, R. The relationship between return and market value of common stock. Journal of Financial Economics, v. 9, n. 1, p. 3-18, 1981.

BARROS, L. de C.; FAMÁ, R.; SILVEIRA, H. P. Aspectos da teoria de portfolio em mercados emergentes: uma análise de aproximações para a taxa livre de risco no Brasil.In: Seminários em Administração (SEMEAD), 6, 2003. Anais... Universidade de São Paulo, São Paulo, 2003.

BASU, S. Investment performance of common stocks in relation to their price earnings ratios: a test of the efficient market hypothesis. Journal of Finance, v. 32, n. 3, p. 663-682, 1977.

BREALEY, R. A.; MYERS, S. C. Principles of corporate finance. 7. ed. New York: McGraw Hill, 2003.

CARHART, M. M. On persistence in mutual fund performance. Journal of Finance, v. 52, n. 1, p. 57-82, 1997.

ELTON, E. J. et al. Moderna teoria de carteiras e análise de investimentos. São Paulo: Atlas, 2004.

FAMA, E. F.; FRENCH, K. The cross-section of expected stock returns. Journal of Finance, v. 47, n. 2, p . 427-465, 1992.

Common risk factors in the returns on stocks and bonds. Journal of Financial Economics, v. 33, n. 1, p. 3-56, 1993.

Multifactor explanation of asset pricing anomalies. Journal of Finance, v. 56, n. 1, p. 55-84, 1996.

The capital asset pricing model: theory and evidence. Journal of

Economic Perspectives, v. 18, n. 3, p. 25-46, 2004.

FAMA, E. F.; MACBETH, J. D. Risk, return and equilibrium: empirical testes.

Journal of Political Economy, v. 81, n. 3, p. 607-636, 1973.

GRINBLATT, M.; TITMAN, S. Mercados financeiros e estratégia corporativa. 2. ed. Porto Alegre: Bookman, 2005.

JEGADEESH, N.; TITMAN, S. Returns to buying winners and selling losers: implications for stock market efficiency. Journal of Finance, v. 48, n. 1, p. 65-91, 1993. 
Profitability of momentum strategies: an evaluation of alternative explanations. Journal of Finance, v. 56, n. 2, p. 699-720, april 2001.

LAKONISHOK, J.; SHAPIRO, A. C. Systematic risk, total risk and size as determinants of stock market returns. Journal of Banking and Finance, v. 10, n. 1, p. 115-132, 1986.

LUCENA, P.; PINTO, A. C. F. Estudo de anomalias no mercado brasileiro de ações através de uma modificação no modelo de Fama e French. In: ENCONTRO ANUAL DA ASSOCIAÇÃO NACIONAL DOS PROGRAMAS DE PÓSGRADUAÇÃO EM ADMINISTRAÇÃO, 29., 2005, Brasília. Anais... Brasília: ANPAD, 2005.

MÁLAGA, F. K.; SECURATO, J. R. Aplicação do modelo dos três fatores de Fama \& French no mercado acionário brasileiro - um estudo empírico do período 1995-2003. In: Encontro Anual da Associação Nacional dos Programas de Pós-graduação em Administração, 28, 2004, Curitiba. Anais...Curitiba: ANPAD, 2004.

MARKOWITZ, H. M. Portfolio Selection. Journal of Finance, v. 7, p. 77-91, 1952.

MUSSA, A. SANTOS, J. O., FAMÁ, R. A Adição do Fator de Risco Momento ao Modelo de Precificação de Ativos dos Três Fatores de Fama \& French, Aplicado ao Mercado Acionário Brasileiro. In: Congresso USP de Controladoria e Contabilidade, 7., 2007, São Paulo. Anais... São Paulo: USP, 2007.

ROSS, S. A. The arbitrage theory of capital asset pricing. Journal of Economic Theory, v. 13, p. 341-360, 1976.

ROUWENHORST, K. G. International momentum estrategies. Journal of Finance, v. 53, n. 1, p. 267-284, feb. 1998.

Local return factors and turnover in emerging stock markets. Journal of Finance, v. 54, n. 4, p. 1439-1464, aug. 1999.

SHARPE, W. F. Capital asset prices: a theory of market equilibrium under conditions of risk. Journal of Finance, v. 19, p. 425-443, 1964.

STATTMAN, D. Book values and stock returns. The Chicago MBA: A Journal of Selected Papers, v. 4, p. 25-45, 1980.

TOBIN, J. Liquidity preference as behavior toward risk. The Review of Economic Studies, v. 25, n. 2, p. 65-86, 1958. 